\title{
Proton therapy for small cell lung cancer
}

\author{
Vivek Verma ${ }^{1}$, J. Isabelle Choi ${ }^{2}$, Charles B. Simone II $^{2}$ \\ ${ }^{1}$ Department of Radiation Oncology, University of Nebraska Medical Center, Omaha, Nebraska, USA; ${ }^{2}$ Department of Radiation Oncology, \\ University of Maryland Medical Center, Baltimore, Maryland, USA \\ Contributions: (I) Conception and design: CB Simone 2nd; (II) Administrative support: CB Simone 2nd; (III) Provision of study materials or patients: \\ None; (IV) Collection and assembly of data: All authors; (V) Data analysis and interpretation: All authors; (VI) Manuscript writing: All authors; (VII) \\ Final approval of manuscript: All authors. \\ Correspondence to: Charles B. Simone II, MD. Department of Radiation Oncology, University of Maryland School of Medicine, Maryland Proton \\ Treatment Center, 850 W. Baltimore St., Baltimore, MD 21201, USA. Email: charlessimone@umm.edu.
}

\begin{abstract}
The prognosis of limited-stage small cell lung cancer (LS-SCLC) continues to improve and is now roughly comparable to that of locally advanced non-small cell lung cancer (NSCLC). This shift, taken together with the decreased toxicities of modern radiotherapy (RT) for LS-SCLC compared with those reported in historical trials, necessitates further evaluation of whether proton beam therapy (PBT) could further reduce both acute and late toxicities for patients receiving concurrent chemoradiotherapy for LSSCLC. These notions are discussed theoretically, with an emphasis on cardiac events. This is followed by a review of the published evidence to date demonstrating improved dosimetry with PBT over intensitymodulated RT and encouraging safety and efficacy profiles seen in early clinical reports. In addition to covering technical aspects of PBT for LS-SCLC such as intensity-modulated PBT, image-guidance for PBT, and adaptive planning, this review also discusses the need for increased data on intensity-modulated PBT for LS-SCLC, economic and quality of life analyses for future PBT SCLC studies, careful categorization of cardiac events in these patients, and the role for immunotherapy combined with photon- or proton-based RT for LS-SCLC.
\end{abstract}

Keywords: Small cell lung cancer (SCLC); limited stage; radiation therapy; proton beam therapy (PBT); toxicities

Submitted Dec 12, 2017. Accepted for publication Mar 28, 2018.

doi: $10.21037 /$ tlcr.2018.04.02

View this article at: http://dx.doi.org/10.21037/tlcr.2018.04.02

\section{Introduction}

Small cell lung cancer (SCLC) comprises a minority of lung cancer diagnoses but displays considerably more aggressive behavior than non-SCLC (NSCLC). Roughly two-thirds of SCLC cases are extensive stage (ES-SCLC), and the remainder have thorax-confined, non-metastatic disease, termed limited-stage SCLC (LS-SCLC). Chemotherapy is a cornerstone of therapy for both LS-SCLC and ESSCLC, while surgery (1) and stereotactic body radiation therapy (2-4) play a role in only the minority of LS-SCLC cases. Although the role of thoracic radiation therapy (RT) continues to evolve for ES-SCLC (5), it is considered crucial to provide satisfactory outcomes in LS-SCLC $(6,7)$.

Prognosis for LS-SCLC has historically been poor, with the median overall survival (OS) previously estimated to be around 1 year (6). Since then, however, OS has substantially increased owing to advancements in imaging-based staging (8), treatment paradigms, and salvage therapies. A pivotal trial that accrued from 1989-1992 observed a median OS of 19 months in the once-daily RT group and 23 months in the twice-daily cohort (7). Survival continues to numerically increase in the most contemporary trials, such as the finding of median OS of 25 and 30 months in the once- and twice-daily arms in the CONVERT trial, respectively, which accrued patients from 2008-2013 (9). Data from CONVERT show the numerically highest $O S$ to date, which are roughly comparable to, or higher than, those in modern trials for locally-advanced NSCLC $(10,11)$. 
The successes of modern combined-modality management have come not only in the form of outcome improvements, but also in toxicity reductions. The latter is critically important, because concurrent high-dose RT and chemotherapy can incur substantial toxicities given patients are often of advanced age with preexisting comorbidities and being treated to large-volume and centrally located malignancies that abut or invade several organs-at-risks (OARs), such as the esophagus, normal lung tissues, and heart. When comparing modern data with threedimensional treatment planning (9) to older trials evaluating similar treatment paradigms but with two-dimensional planning (7), the former has resulted in lower rates of highgrade adverse events.

As radiotherapeutic technology has continued to advance, there have been ongoing attempts to improve the therapeutic ratio of cancer therapy. The rapid adoption and utilization of inverse-planned intensity-modulated RT (IMRT) represents a major achievement in this realm. IMRT results in highly conformal dose distributions that better spare OARs as compared with three-dimensional conformal RT (3DCRT). Although the value of IMRT is just beginning to be proven in the prospective setting for NSCLC (12), its utility for LS-SCLC is even less understood, as exemplified by just $16-17 \%$ of patients receiving IMRT in the modern CONVERT trial (9). Accordingly, there are only a few published reports of concurrent IMRT and chemotherapy for LS-SCLC, and while these show promising outcomes and toxicity profiles, they are as of yet uncorroborated by high-volume prospective data (13-16).

Proton beam therapy (PBT) represents the most advanced RT modality for oncologic management. The proton particle is associated with a selective energy deposition known as the Bragg peak, beyond which there is no deposited dose (17). As a result, an advantage of proton therapy over photon-based techniques such as IMRT lies in that the former can generally be accomplished with fewer RT beams, notably reducing the areas receiving low RT doses and decreasing the integral dose of irradiation received by normal tissues. Based on available clinical data, PBT displays promising outcomes and toxicity profiles for several neoplasms (18-23), including intrathoracic malignancies (24-32).

\section{Rationale for PBT in LS-SCLC}

The life expectancy for LS-SCLC continues to rise, which parallels that of locally advanced NSCLC in many respects. With regard to the latter, a major lesson learned from the Radiation Therapy Oncology Group (RTOG) 0617 trial was the association between cardiac irradiation doses and survival. Historically, cardiotoxicity (which had been generally categorized as a late event) was not a major consideration for treatment planning in LA-NSCLC (33). However, the substantially improved prognosis of these patients, together with the recognition that cardiotoxicity may have a more acute/subacute natural history than initially hypothesized, necessitates a revision of established dogma (34-36).

Cardiac-sparing conformal RT is beginning to be performed in locally advanced NSCLC but has not yet commenced in LS-SCLC. Hence, it may be logically postulated that, with the numerically similar survival observed in contemporary trials of each of these types of lung cancer $(9,11)$, cardiac doses are of prime importance in both neoplasms. As studies examining dose-volume relationships of the heart and cardiac events in patients with LS-SCLC have heretofore not been performed, this remains a prime area for future investigation. To this extent, highly conformal RT such as PBT offers great potential to spare the heart (37) and thus potentially reduce morbidities and even mortality, if the results of RTOG 0617 may be extrapolated to LS-SCLC.

Additionally, many patients with LS-SCLC have significant smoking histories and other comorbidities, making the challenging regimen of full-dose RT concurrently with chemotherapy even more difficult to tolerate without treatment breaks or dose reductions. Thus, strategies to reduce acute adverse events are critical and may result in enhanced tolerability of curative-intent concurrent chemoradiotherapy. Doing so may maximize outcomes, as suboptimal chemotherapy receipt and RT treatment breaks correlate with poorer prognoses in lung cancer $(38,39)$. Furthermore, because patients with LS-SCLC can develop locoregional recurrence following chemoradiotherapy, PBT may also allow for safer reirradiation if clinically warranted (40).

Lastly, PBT for locally advanced NSCLC is buoyed by analyses demonstrating it to be a relatively cost-effective measure that produces favorable quality of life over photon-based techniques (41-43). If these notions may also be extrapolated to LS-SCLC, which often presents with bulkier disease closer to dose-limiting mediastinal structures, it is also possible that PBT for LS-SCLC may display similarly encouraging economic and quality of life profiles. 


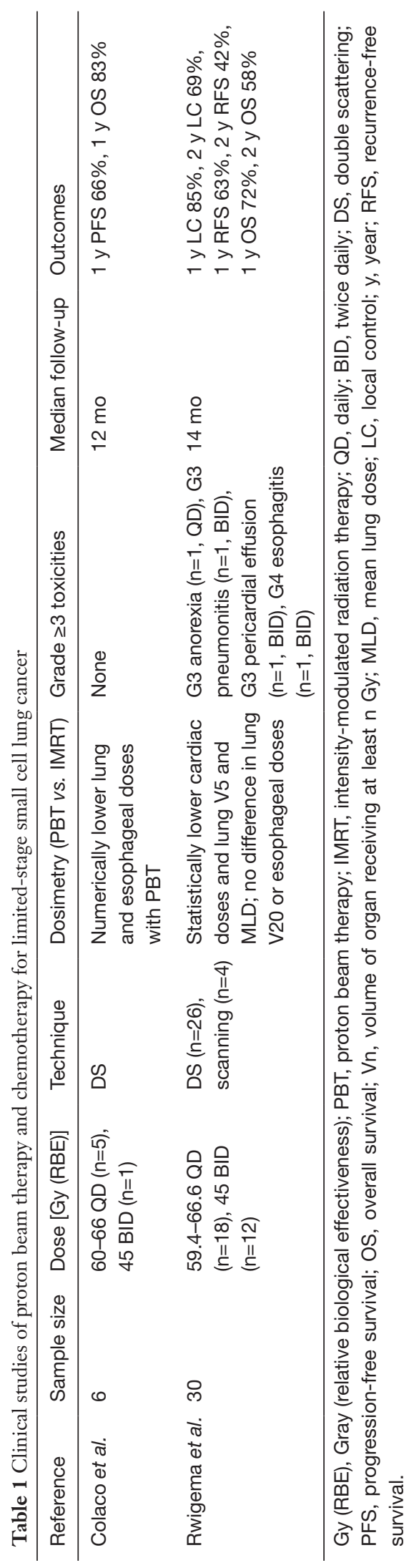

\section{Clinical evidence}

To date, there are two known clinical publications of PBT for LS-SCLC (Table 1). ES-SCLC will not be discussed further in this review, as the poor prognosis, together with lower thoracic irradiation doses typically employed, mute some of the potential advantages of PBT in this population.

Colaco and colleagues at the University of Florida published a 6-patient series of PBT and cisplatin/ etoposide (44). All but one patient received concurrent therapy, and all but one patient also received daily RT [60-66 Gy (relative biological effectiveness (RBE)]. Dosimetric analysis of lung and esophageal doses of PBT as compared with backup IMRT plans showed numerical superiority for the former (no statistics were performed), most apparent with low-dose parameters. At a median follow-up of 1 year, the progression-free survival and OS were $66 \%$ and $83 \%$, respectively. No patient experienced grade $\geq 3$ acute or late events.

The largest report of 30 patients was recently published as part of a prospective observational study at the University of Pennsylvania (45). All patients received a platinum agent and etoposide concurrently with PBT. Twelve patients received twice-daily PBT [45 Gy (RBE)], and the remainder underwent daily treatment [59.4-66.6 Gy (RBE)]. Dosimetric analyses revealed multiple statistically lower heart dose parameters along with reductions in lung V5 and mean lung doses (but not V20) with proton therapy. Grade 3 toxicities were limited to one case of anorexia (daily treatment) and one case each of pneumonitis and pericardial effusion (twice daily treatment). One grade 4 case of esophagitis occurred in a patient receiving twice daily PBT. At median follow-up of 14 months, the 1 and 2-year local control, recurrence-free survival, and OS were $85 \%$ and $69 \%, 63 \%$ and $42 \%$, and $72 \%$ and $58 \%$, respectively. Five patients recurred in-field, two of which were isolated infield failures.

Taken together, both studies show encouraging safety and efficacy profiles, numerically comparable or favorable as compared to Intergroup 0096 and CONVERT data (45). Although the study from University of Florida investigators did not assess heart dose, the prospective study from University of Pennsylvania investigators demonstrated dosimetric improvements in cardiac V30, $\mathrm{V} 45$, and mean doses with protons. It also exemplified the aforementioned notion of combined modality treatment tolerance, as no patient required a RT break and 93\% of patients completed all four cycles of chemotherapy. 
It should also be emphasized that PBT displayed these outcomes in light of potential biases in patient selection (for inclusion into prospective studies, or retrospective studies), namely, that patients with large-volume disease and/or disease more intimately involving OARs are often deemed potentially unsafe for photon therapy and may have been more likely treated with PBT (or enrolled onto prospective PBT trials). This is especially important considering that IMRT is inverse-planned, whereas the vast majority of patients in both series received forward-planned, passively scattered PBT. Also, patients with insurance plans more likely to cover PBT (e.g., Medicare) could comprise large proportions of these studies, but the Medicare population also tends to be older with a potentially higher risk of therapy-induced toxicities (46).

\section{Technical aspects}

As a relatively new technology, PBT for LS-SCLC is technically challenging to treat for several reasons. First, as mentioned above, the use of forward-planned PBT alone does not guarantee higher conformality over inverseplanned IMRT, especially for bulky mediastinal disease in close proximity to multiple OARs (30). This could have caused the lack of significant differences in esophageal dosimetry as observed by Rwigema et al. (45). Moreover, accounting for range uncertainties in treatment planning often necessitates the use of an additional margin, which is not analogously performed in photon-based treatment planning. Additionally, because the majority of PBT machines worldwide are not equipped with kilovoltage cone-beam computed tomography (47), the resulting use of relatively generous margins may also lead to treatment of larger volumes than otherwise necessary.

The aforementioned clinical findings $(44,45)$ must also be contextualized by the small minority of patients who received scanning proton beams in these studies. All patients in the Florida series received passively scattered PBT, along with 26 of 30 patients in the prospective study. Inverse-planned PBT, also known as intensity-modulated proton therapy (IMPT), is a highly conformal technique (more so than forward-planned PBT) that can better spare normal tissues at risk and is more appropriately compared to inverse-planned IMRT (48). Although just a few centers across the world have implemented IMPT to treat thoracic malignancies, the number is expected to rise sharply in the future. As such, results of IMPT as part of combinedmodality therapy for LS-SCLC must be reported and roughly compared to existing data to evaluate differential clinical toxicity reductions with IMPT over forwardplanned PBT.

SCLC is a neoplasm that is well known to demonstrate an excellent response to chemotherapy and/or RT, often leading to major tumor regression as early as 1-2 weeks into therapy commencement. This poses a major challenge for PBT, because replacement of neoplastic (higher density) tissue with lung tissue (lower density) can cause misdistributions in proton dose, resulting in unwanted irradiation delivered to areas such as normal (or contralateral) lung or overshooting of dose into the mediastinum and heart (37). As a result, adaptive therapy (re-simulation and re-planning based on tumor response or other anatomical changes during treatment) may be necessary when delivering PBT. With regard to LSSCLC, just three patients $(10 \%)$ in the University of Pennsylvania investigation required adaptive re-planning. These notions are similar to principles of why photonbased RT is most often given after completion of the first cycle of chemotherapy (i.e., to avoid treating large lung volumes), along with necessitating re-planning for higher conformality techniques that are better suited to spare more normal tissue.

\section{Future directions}

Although PBT utilization for LS-SCLC is still in its infancy, it remains an attractive technique for several reasons, in particular possible reductions in radiation-induced cardiac damage in patients who are now experiencing numerically prolonged survival and increasing chance of cure. Further evidence to justify this modality for LS-SCLC is critical, including economic and quality of life analyses.

Despite the growing use of PBT worldwide, the few data in LS-SCLC may in part be attributed to the lack of consensus definitively establishing IMRT as the technique of choice for these patients. Clearly, however, with newer high-quality data supporting the role of IMRT for NSCLC (12), attention to advanced RT modalities should shift to LS-SCLC as well. Thus, demonstrating encouraging outcomes and toxicities for IMRT in LSSCLC may serve as a "bridge" to further investigation of PBT in this population.

Moving forward, studies should look to carefully define cardiac events with long-term follow-up, especially in patients with pre-existing comorbidities versus lack thereof. With precise categorization of these toxicities, 
Table 2 Accruing clinical trials of immunotherapy for small cell lung cancer

\begin{tabular}{|c|c|c|c|c|c|c|}
\hline NCT number & Title & Institution(s) & Phase & Stage & $\begin{array}{l}\text { Estimated } \\
\text { accrual }\end{array}$ & Primary endpoint(s) \\
\hline 03059667 & $\begin{array}{l}\text { Immunotherapy as second-line in patients with } \\
\text { small cell lung cancer }\end{array}$ & $\begin{array}{l}\text { French } \\
\text { Intergroup }\end{array}$ & II & LS/ES & 70 & $\begin{array}{l}\text { Response rate to } \\
\text { atezolizumab }\end{array}$ \\
\hline 01840579 & $\begin{array}{l}\text { Study of pembrolizumab (MK-3475) monotherapy } \\
\text { in advanced solid tumors and pembrolizumab } \\
\text { combination therapy in advanced non-small cell } \\
\text { lung cancer/extensive-disease small cell lung } \\
\text { cancer }\end{array}$ & $\begin{array}{l}\text { Japan, multi- } \\
\text { institutional }\end{array}$ & I & ES & 75 & Dose-limiting toxicities \\
\hline 02538666 & $\begin{array}{l}\text { An investigational immunotherapy study } \\
\text { of nivolumab, or nivolumab in combination } \\
\text { with ipilimumab, or placebo in patients with } \\
\text { extensive-stage disease small cell lung cancer } \\
\text { (ED-SCLC) after completion of platinum-based } \\
\text { chemotherapy }\end{array}$ & $\begin{array}{l}\text { USA, multi- } \\
\text { institutional }\end{array}$ & III & ES & 810 & OS, PFS \\
\hline
\end{tabular}

NCT, National Clinical Trials (registry); LS, limited stage; ES, extensive stage; OS, overall survival; PFS, progression-free survival.

reliable dose-volume parameters (with regard to various substructures of the heart) can eventually be generated. This is especially important for LS-SCLC patients in light of the CONVERT trial; despite showing no differences in outcomes, cardiotoxicities could be less in patients treated twice daily, which delivers $25-35 \%$ less overall dose to the heart than daily, higher dose treatment.

Finally, the rise of immunotherapy for oncologic management is also being investigated for LS-SCLC; a search of ongoing clinical trials involving immunotherapy for SCLC is shown in Table 2. In addition, although the immunogenicity of SCLC remains under investigation, combining RT with immunotherapy may serve to better optimize the immunogenicity of lung cancer $(49,50)$. Furthermore, there are data suggesting that proton or heavy ions may stimulate the immune system to a greater degree than photons $(51,52)$, potentially further maximizing any synergistic effects of RT and immunotherapy for SCLC. Unfortunately, safety data for concurrent immunotherapy and RT is largely lacking at present. Given this lack of clinical evidence, continued reporting of institutional series is necessary to make more firm conclusions than those that exist currently, and prospective trials are encouraged.

\section{Acknowledgements}

None.

\section{Footnote}

Conflicts of Interest: The authors have no conflicts of interest to declare.

\section{References}

1. Inoue M, Miyoshi S, Yasumitsu T, et al. Surgical results for small cell lung cancer based on the new TNM staging system. Thoracic Surgery Study Group of Osaka University, Osaka, Japan. Ann Thorac Surg 2000;70:1615-9.

2. Verma V, Simone CB 2nd, Zhen W. Stereotactic Radiotherapy for Stage I Small Cell Lung Cancer. Oncologist 2016;21:131-3.

3. Verma V, Simone CB 2nd, Allen PK, et al. MultiInstitutional Experience of Stereotactic Ablative Radiation Therapy for Stage I Small Cell Lung Cancer. Int J Radiat Oncol Biol Phys 2017;97:362-71.

4. Verma V, Simone CB 2nd, Allen PK, et al. Outcomes of Stereotactic Body Radiotherapy for T1-T2N0 Small Cell Carcinoma According to Addition of Chemotherapy and Prophylactic Cranial Irradiation: A Multicenter Analysis. Clin Lung Cancer 2017;18:675-81.e1.

5. Slotman BJ, van Tinteren H, Praag JO, et al. Use of thoracic radiotherapy for extensive stage small-cell lung cancer: a phase 3 randomised controlled trial. Lancet 2015;385:36-42. 
6. Pignon JP, Arriagada R, Ihde DC, et al. A meta-analysis of thoracic radiotherapy for small-cell lung cancer. N Engl J Med 1992;327:1618-24.

7. Turrisi AT 3rd, Kim K, Blum R, et al. Twice-daily compared with once-daily thoracic radiotherapy in limited small-cell lung cancer treated concurrently with cisplatin and etoposide. N Engl J Med 1999;340:265-71.

8. Xanthopoulos EP, Corradetti MN, Mitra N, et al. Impact of PET staging in limited-stage small-cell lung cancer. J Thorac Oncol 2013;8:899-905.

9. Faivre-Finn C, Snee M, Ashcroft L, et al. Concurrent once-daily versus twice-daily chemoradiotherapy in patients with limited-stage small-cell lung cancer (CONVERT): an open-label, phase 3, randomised, superiority trial. Lancet Oncol 2017;18:1116-25.

10. Suntharalingam M, Paulus R, Edelman MJ, et al. RTOG 0229: A Phase II Trial of Neoadjuvant Therapy with Concurrent Chemotherapy and Full Dose Radiotherapy (XRT) followed by Resection and Consolidative Therapy for LA-NSCLC. Int J Radiat Oncol Biol Phys 2010;78:S111.

11. Bradley JD, Paulus R, Komaki R, et al. Standard-dose versus high-dose conformal radiotherapy with concurrent and consolidation carboplatin plus paclitaxel with or without cetuximab for patients with stage IIIA or IIIB non-small- cell lung cancer (RTOG 0617): a randomised, two-by- two factorial phase 3 study. Lancet Oncol 2015;16:187-99.

12. Chun SG, Hu C, Choy H, et al. Impact of IntensityModulated Radiation Therapy Technique for Locally Advanced Non-Small-Cell Lung Cancer: A Secondary Analysis of the NRG Oncology RTOG 0617 Randomized Clinical Trial. J Clin Oncol 2017;35:56-62.

13. Shirvani SM, Komaki R, Heymach JV, et al. Positron emission tomography-guided intensity modulated radiotherapy for limited-stage small-cell lung cancer. Int J Radiat Oncol Biol Phys 2012;82:e91-7.

14. Shirvani SM, Juloori A, Allen PK, et al. Comparison of 2 Common Radiation Therapy Techniques for Definitive Treatment of Small Cell Lung Cancer. Int J Radiat Oncol Biol Phys 2013;87:139-47.

15. Xia B, Hong LZ, Cai XW, et al. Phase 2 Study of Accelerated Hypofractionated Thoracic Radiation Therapy and Concurrent Chemotherapy in Patients With LimitedStage Small-Cell Lung Cancer. Int J Radiat Oncol Biol Phys 2015;91:517-23.

16. Liu Z, Liu W, Ji K, et al. Simultaneous integrated dose reduction intensity-modulated radiotherapy applied to an elective nodal area of limited-stage small-cell lung cancer. Exp Ther Med 2015;10:2083-7.

17. Patyal B. Dosimetry aspects of proton therapy. Technol Cancer Res Treat 2007;6:17-23.

18. Verma V, Mehta MP. Clinical outcomes of proton radiotherapy for uveal melanoma. Clin Oncol (R Coll Radiol) 2016;28:e17-27.

19. Verma V, Shah C, Mehta MP. Clinical outcomes and toxicity of proton radiotherapy for breast cancer. Clin Breast Cancer 2016;16:145-54.

20. Verma V, Lin SH, Simone CB 2nd, et al. Clinical outcomes and toxicities of proton radiotherapy for gastrointestinal neoplasms: a systematic review. J Gastrointest Oncol 2016;7:644-64.

21. Verma V, Simone CB 2nd, Wahl AO, et al. Proton radiotherapy for gynecologic neoplasms. Acta Oncol 2016;55:1257-65.

22. Verma V, Iftekaruddin Z, Badar N, et al. Proton beam radiotherapy as part of comprehensive regional nodal irradiation for locally advanced breast cancer. Radiother Oncol 2017;123:294-8.

23. Adeberg S, Bernhardt D, Harrabi SB, et al. Sequential proton boost after standard chemoradiation for high-grade glioma. Radiother Oncol 2017;125:266-72.

24. Chuong MD, Hallemeier CL, Jabbour SK, et al. Improving outcomes for esophageal cancer using proton beam therapy. Int J Radiat Oncol Biol Phys 2016;95:488-97.

25. Verma V, Moreno AC, Lin SH. Advances in radiotherapy management of esophageal cancer. J Clin Med 2016;5:E91.

26. Lin SH, Merrell KW, Shen J, et al. Multi-institutional analysis of radiation modality use and postoperative outcomes of neoadjuvant chemoradiation for esophageal cancer. Radiother Oncol 2017;123:376-81.

27. Chang JY, Verma V, Li M, et al. Proton Beam Radiotherapy and Concurrent Chemotherapy for Unresectable Stage III Non-Small Cell Lung Cancer: Final Results of a Phase 2 Study. JAMA Oncol 2017;3:e172032.

28. Simone CB 2nd, Rengan R. The use of proton therapy in the treatment of lung cancers. Cancer J 2014;20:427-32.

29. Kesarwala AH, Ko CJ, Ning H, et al. Intensity- modulated proton therapy for elective nodal irradiation and involvedfield radiation in the definitive treatment of locally advanced non-small-cell lung cancer: a dosimetric study. Clin Lung Cancer 2015;16:237-44.

30. Chang JY, Jabbour SK, De Ruysscher D, et al. Consensus statement on proton therapy in early-stage and locally advanced non-small cell lung cancer. Int J Radiat Oncol 
Biol Phys 2016;95:505-16.

31. Giaddui T, Chen W, Yu J, et al. Establishing the feasibility of the dosimetric compliance criteria of RTOG 1308: phase III randomized trial comparing overall survival after photon versus proton radiochemotherapy for inoperable stage II-IIIB NSCLC. Radiat Oncol 2016;11:66.

32. Higgins KA, O'Connell K, Liu Y, et al. National Cancer Database Analysis of Proton Versus Photon Radiation Therapy in Non-Small Cell Lung Cancer. Int J Radiat Oncol Biol Phys 2017;97:128-37.

33. Haque $W$, Verma V, Fakhreddine $M$, et al. Trends in Cardiac Mortality in Patients with Locally Advanced NonSmall Cell Lung Cancer. Int J Radiat Oncol Biol Phys 2018;100:470-7.

34. Simone CB 2nd. New era in radiation oncology for lung cancer: recognizing the importance of cardiac irradiation. J Clin Oncol 2017;35:1381-3.

35. Simone CB 2nd. Reply to J.P. Gross et al. J Clin Oncol 2017;35:2216-7.

36. Verma V, Simone CB 2nd, Werner-Wasik M. Acute and Late Toxicities of Concurrent Chemoradiotherapy for Locally-Advanced Non-Small Cell Lung Cancer. Cancers (Basel) 2017;9:120.

37. Lu D, Xanthopoulos E, Dixit N, et al. Comparison of Intensity Modulated Radiotherapy, Adaptive Radiotherapy, Proton Radiotherapy, and Adaptive Proton Radiotherapy for Small Cell Lung Cancer. Appl Radiat Oncol 2016;5:20-7.

38. Karim SM, Zekri J. Chemotherapy for small cell lung cancer: a comprehensive review. Oncol Rev 2012;6:e4.

39. McMillan MT, Ojerholm E, Verma V, et al. Radiation Treatment Time and Overall Survival in Locally Advanced Non-small Cell Lung Cancer. Int J Radiat Oncol Biol Phys 2017;98:1142-52.

40. Verma V, Rwigema JM, Malyapa RS, et al. Systematic assessment of clinical outcomes and toxicities of proton radiotherapy for reirradiation. Radiother Oncol 2017;125:21-30.

41. Verma V, Mishra MV, Mehta MP. A systematic review of the cost and cost-effectiveness studies of proton radiotherapy. Cancer 2016;122:1483-501.

42. Verma V, Shah C, Rwigema JC, et al. Cost-

Cite this article as: Verma V, Choi JI, Simone CB 2nd. Proton therapy for small cell lung cancer. Transl Lung Cancer Res 2018;7(2):134-140. doi: 10.21037/tlcr.2018.04.02 comparativeness of proton versus photon therapy. Chin Clin Oncol 2016;5:56.

43. Verma V, Simone CB 2nd, Mishra MV. Quality of life and patient-reported outcomes following proton radiation therapy: a systematic review. J Natl Cancer Inst 2018;110(4).

44. Colaco RJ, Huh S, Nichols RC, et al. Dosimetric rationale and early experience at UFPTI of thoracic proton therapy and chemotherapy in limited-stage small cell lung cancer. Acta Oncol 2013;52:506-13.

45. Rwigema JM, Verma V, Lin L, et al. Prospective study of proton-beam radiation therapy for limited-stage small cell lung cancer. Cancer 2017;123:4244-51.

46. Verma V, Rwigema JM, Adeberg S, et al. Enrollment of Elderly Patients With Locally Advanced Non-Small Cell Lung Cancer in Multi-institutional Trials of Proton Beam Radiation Therapy. Clin Lung Cancer 2017;18:441-3.

47. Veiga C, Janssens G, Teng CL, et al. First Clinical Investigation of Cone Beam Computed Tomography and Deformable Registration for Adaptive Proton Therapy for Lung Cancer. Int J Radiat Oncol Biol Phys 2016;95:549-59.

48. Lin L, Kang M, Huang S, et al. Beam-specific planning target volumes incorporating 4D CT for pencil beam scanning proton therapy of thoracic tumors. J Appl Clin Med Phys 2015;16:5678.

49. Simone CB 2nd, Burri SH, Heinzerling JH. Novel radiotherapy approaches for lung cancer: combining radiation therapy with targeted and immunotherapies. Transl Lung Cancer Res 2015;4:545-52.

50. Simone CB 2nd, Berman AT, Jabbour SK. Harnessing the potential synergy of combining radiation therapy and immunotherapy for thoracic malignancies. Transl Lung Cancer Res 2017;6:109-12.

51. Gameiro SR, Malamas AS, Bernstein MB, et al. Tumor Cells Surviving Exposure to Proton or Photon Radiation Share a Common Immunogenic Modulation Signature, Rendering Them More Sensitive to T Cell-Mediated Killing. Int J Radiat Oncol Biol Phys 2016;95:120-30.

52. Shimokawa T, Ma L, Ando K, et al. The Future of Combining Carbon-Ion Radiotherapy with Immunotherapy: Evidence and Progress in Mouse Models. Int J Particle Ther 2016;3:61-70. 